\title{
ESTILO DE VIDA Y AUTOESTIMA EN PERSONAS DIABÉTICAS DE UNA COMUNIDAD SUBURBANA
}

\begin{abstract}
LIFESTYLE AND SELF-ESTEEM IN PERSONS WITH DIABETES IN A SUBURBAN COMMUNITY
\end{abstract}

Recibido 16 diciembre 2014 Aceptado 26 de febrero 2015

Correspondencia: Sofía Cheverría-Rivera Maestría en Administración de la Atención de Enfermería. Facultad de Enfermería, UASLP sofia@uslp.mx

\section{Autores:}

Sofía Cheverría-Rivera.

Maestría en Administración de la Atención de Enfermería.

Facultad de Enfermería, UASLP

Martha Landeros-López.

Doctorado en Enfermería. Facultad de Enfermería.

Facultad de Enfermería, UASLP

María Candelaria Betancourt-Esparza.

Doctorado en Enfermería. Facultad de Enfermería.

Facultad de Enfermería, UASLP

Rina Orozco-Medina.

Estudiante de Licenciatura en Gerontogeriatría

Universidad de Guadalajara

Raúl Hernández-Cruz.

Estudiante de Maestría en Administración de la Atención de Enfermería, UASLP

Palabras clave: Estilo de vida, autoestima, Diabetes Mellitus tipo 2, conductas saludables, adulto, anciano.

Key Words: Life Style, Self Concept, Diabetes Mellitus, Health Behavior, Adult, Aged. 


\section{RESUMEN}

Introducción. Los estilos de vida y la autoestima repercuten en las conductas de salud de la persona con Diabetes Mellitus tipo 2 (DM2) para mantener un control.

Objetivo: Identificar la relación entre estilo de vida y autoestima de personas DM2.

Material y métodos: Estudio descriptivo-transversal y correlacional en 81 personas de una comunidad suburbana en San Luis Potosí, México. Se incluyeron adultos y adultos mayores, ambos sexos con DM2 que aceptaron participar. Fueron aplicados: a) Instrumento para medir el estilo de vida en diabéticos (IMEVID) con 25 tems que pondera como favorable, poco favorable y desfavorable); b) Escala de autoestima con 10 ítems (muy de acuerdo a muy en desacuerdo) ponderándose alta, media y baja. El procesamiento y análisis fue en SPSS versión 20, se obtuvo estadística descriptiva y asociación mediante correlación de Pearson.

Resultados: La media de edad fue 57.9 años, y predominio femenino. La autoestima alta se encontró en $62.9 \%$ y el estilo de vida en $51.7 \%$ de poco favorable a desfavorable, estadísticamente no se asociaron como en otros estudios.

Conclusiones: Es necesario promover el estilo de vida favorable, y aunque no se asociaron las variables, la autoestima media-alta es una fortaleza para el control de la enfermedad.

Palabras clave: Estilo de vida, autoestima, Diabetes Mellitus tipo 2, conductas saludables, adulto, anciano.

\section{ABSTRACT}

Introduction. Lifestyles and self-esteem affect health behaviors of people with Diabetes Mellitus type 2 (DM2) to maintain control.

Objective. To identify the relation between lifestyles and the self-esteem, in diabetics type 2.

Material and methods: Descriptive- transversal and Correlational study, in 81 persons from a suburban community in San Luis Potosí, Mexico. Adults and seniors, men and women with DM2 who agreed to participate were included. Were applied: a) An instrument for measuring the lifestyle in diabetic (IMEVID) with 25 items that weights be favorable, unfavorable and favorable); b) selfesteem scale with 10 items (strongly agree to strongly disagree) weighted high, medium and low. The processing and analysis was SPSS version 20, descriptive and statistical association was obtained by Pearson correlation.

Results: The mean age was 57.9 years, and female predominance. High self-esteem was found in $62.9 \%$ and lifestyle $57.7 \%$ from unfavorable to favorable, were not associated statistically as in other studies.

Conclusions: It is necessary to promote favorable lifestyle, and although the variables were not associated, medium-high self-esteem is a strength to control the disease.

Key Words: Life Style, Self Concept, Diabetes Mellitus, Health Behavior, Adult, Aged.

\section{0}

nMNMMun vol.14. No. 1 enero-abril 2015 


\section{INTRODUCCIÓN}

as enfermedades crónico-degenerativas constituyen la principal causa de muerte a nivel mundial!' La Diabetes Mellitus (DM) está incluida en este grupo de enfermedades y - se calcula que para el 2035 la cifra de personas con este problema aumentará hasta los 592 millones a nivel mundial². En México la DM, en el grupo de 20 a 65 años se encuentra en el primer lugar de causas de defunción con 14.6\%, y en segundo lugar con 15.4\% en el grupo de 66 y más 3.

Según algunos estudios, 5,6, la aparición de la enfermedad puede reducirse de manera significativa mediante la adopción de comportamientos saludables en los estilos de vida, y en la disminución de los factores de riesgo modificables como el sobrepeso u obesidad, y la falta de actividad física. Sin embargo un factor de riesgo no modificable que se encuentra detrás del aumento desenfrenado de la DM son las cuestiones genéticas?.

La enfermedad no solo afecta la salud física de la persona, es una condición que requiere cambios en el estilo de vida, influyendo en el funcionamiento psicológico hasta afectar la autoestima de quienes la padecen. La autoestima según Rosenberg?. se refiere a sentimientos de valía personal y de respeto a sí mismo, y está constituida por factores comportamentales y motivacionales, como el aprendizaje, las creencias y las influencias sociales, que pueden repercutir directamente en las conductas y hábitos que caracterizan el estilo de vida de una persona como la alimentación, la actividad física, adicciones, responsabilidad para la salud y las creencias. Conforme a estos planteamientos el objetivo del estudio fue identificar la relación entre el estilo de vida y la autoestima en personas con DM2 de una comunidad suburbana en San Luis Potosí, México.

\section{MATERIAL Y MÉTODOS}

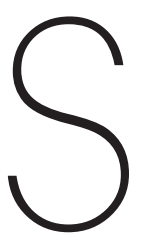

e realizó un estudio observacional con diseño descriptivo-transversal correlacional en el año de 2013 en una comunidad de Villa de Pozos en San Luis Potosí, México. La muestra por conveniencia fue de 81 personas usuarias de los servicios de salud de la Unidad de Cuidados Integrales y de Investigación en Salud (UCIIS), perteneciente a la Universidad Autónoma de San Luis Potosí. Fueron incluidos pacientes diagnosticados con DM2, con edad de adulto y adulto mayor, de ambos sexos y que aceptaron participar voluntariamente bajo consentimiento informado. Los instrumentos aplicados fueron: a) Instrumento para medir el estilo de vida en diabéticos (IMEVID) ${ }^{10}$ cuestionario específico, estandarizado, global y autoadministrado, que tuvo la finalidad de saber y medir el estilo de vida en los pacientes con DM2 de una manera rápida y sencilla; evaluó aspectos de nutrición, actividad física, toxicomanías, orientación e información sobre la enfermedad y aspectos emocionales. Conformado por 25 ítems divididos en 7 dominios con escala de respuesta Likert de tres opciones (0, 2 y 4, este último valor es el más favorable); el puntaje en el rango de 100-75 corresponde a un estilo de vida favorable, y menos de 49 puntos a uno desfavorable.

b) Escala de autoestima de Rosemberg," compuesta por 10 ítems, evaluó el sentimiento de satisfacción que la persona tiene de sí misma a través de una escala de medición de cuatro opciones (de A hasta a D, este último con el mayor valor). El puntaje de 30-40 indica autoestima elevada y menos de 25 la considera baja.

Para el procesamiento y análisis se realizó mediante el SPSS ${ }^{\circledR}$ (Statistical Package for the Social Sciences) versión 20. Se obtuvo estadística descriptiva (frecuencias absolutas y relativas), la asociación entre estilos de vida y autoestima fue analizada con el Coeficiente de correlación de Pearson. 


\section{RESULTADOS}

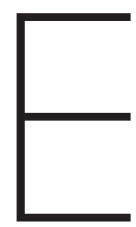

n las 81 personas con diagnóstico de Diabetes Mellitus tipo 2 predominó el sexo femenino (69.1\%); respecto a la edad, la media se ubicó en 57.1 años, mínima de 38 y máxima de 76 años, con Desviación estándar de 8.43; en el rango de 50 a 59 años se ubicó el 48.7\% de las personas.

El estilo de vida de los participantes según niveles identificados se muestran en el Cuadro I. donde se observó que predominó el poco favorable.

Los dominios de estilo de vida según las conductas del paciente, se muestran en el Cuadro II.

Se encontró que los indicadores calificados como favorables fueron actividad física con $45.6 \%$ y relaciones con familia y amistades con 43.2\%, en el nivel de poco favorable se encontraron los indicadores de nutrición con $41.9 \%$ y adherencia al tratamiento con un porcentaje de 38.2\% y el indicador de toxicomanías con 58\% en el nivel de estilo de vida desfavorable (Cuadro III). Dentro del indicador de nutrición la población aludió que entre los alimentos que consumen las frutas las comen todos los días (48.1\%), tortillas consumen de 4 a 6 por día (45.7\%) y en cuanto a si agregan azúcar a sus alimentos el 60.5\% casi nunca lo hace.

El Nivel de autoestima de los pacientes fue alto con 62.9\% (Cuadro IV). Y respecto a la autoestima por sexo, se identificó más alta en las mujeres con un 40.7\% en contraste con la de los hombres. Al analizar el nivel de autoestima por rango de edad se encontró que la autoestima elevada se ubica en el grupo de edad de 50 a 59 años y 60 a 69 años con un 24.6\% cada uno, y una autoestima baja en el rango de edad de 50 a 59 años con un 9.8\%.

Finalmente se encontró que la relación entre los estilos de vida y la autoestima no fue estadísticamente significativa dado un valor de $R^{2}=0.0006$.

\section{DISCUSIÓN}

$\square$ n la muestra conformada por 81 personas se observó un porcentaje de edad con una frecuencia mayor en el sexo femenino donde este hecho lo señalan diversos estudios $5,8,12$. En la muestra se manifiesta una generalidad de población por grupo de edad de 50 a 59 años; así como refirió en el 2013 la Federación Internacional de Diabetes (IDF) en el cual el mayor número de personas con diabetes se encuentra en un rango de 40 a 59 años de edad; sin embargo existe poca diferencia entre los sexos de acuerdo a la cifra mundial de personas con diabetes, tanto en 2013 como la estimación que se hace para el 20307. Este aumento está asociado al desarrollo económico, el envejecimiento de la población, la creciente urbanización, los cambios en la dieta, la poca actividad física y las modificaciones en diferentes patrones de estilo de vida?

Respecto a los resultados, se utilizó para la recolección de información sobre estilo de vida el cuestionario IMEVID' ${ }^{10}$, en el cual se revela que el nivel de estilo de vida de los pacientes encuestados es favorable concordando a los resultados de un estudio previo en la Unidad Básica de Comuneros, Cucutua en $2010^{13}$ en el que el indicador de nutrición se encontró que los pacientes presentaron estilos de vida saludables; en ese estudio se descubrió que el indicador de nutrición predomina con porcentaje favorable y casi en su misma proporción poco favorable y desfavorable, donde se manifiestan resultados favorables de actividad física, relaciones con la familia y amistades; estos indicadores puntualizan ser una influencia importante en adherencia al tratamiento y control en las personas con diabetes, lo anterior confirma en diversos estudios que las relaciones interpersonales de tal forma son determinantes en las personas contar con una red de apoyo familiar para poder sobrellevar el padecimiento de la enfermedad ${ }^{14}$ 
Por otra parte, en el nivel de estilo de vida poco favorable se encontraron los indicadores de nutrición, adherencia al tratamiento y toxicomanías ciertos resultados son similares a los obtenidos en un estudio de investigación en el que determinaron el estilo de vida actual de los pacientes con DM26, en el cual las personas reconocen que no están cumpliendo con las indicaciones de nutrición y toma de medicamentos; lo que representa falla en el cumplimiento del tratamiento por parte de la persona.

De acuerdo a los indicadores que destacaron en este nivel de estilo de vida; nutrición, adherencia al tratamiento y toxicomanías, constituyen tres componentes básicos y fundamentales en la vida del paciente con DM2, los cuales indican el valor del seguimiento de una dieta y ejercicio son la base esencial de un plan terapéutico en conjunto con la adopción de cambios comportamentales, lo que compone un proceso activo en la adherencia terapéutica.

En continuación a lo anterior surgen las influencias sobre las características de la personalidad en los estilos de vida saludables los cuales ejercen una importante función hacia las conductas nocivas para la salud como son la ausencia de conductas de prevención. ${ }^{15}$ Cabe resaltar que la salud física a cualquier edad y particularmente en la etapa adulta y vejez, es el resultado de diversos factores entre los que se incluye la vulnerabilidad hacia ciertas enfermedades, factores ambientales, sociales, entre los cuales se incluye la dieta, estilos de vida, factores psicológicos, patrones de comportamiento ${ }^{15}$ y rasgos de personalidad en donde se desarrollan las habilidades para poseer la autoestima.

En cuanto a los estilos de vida y autoestima consultados en otros estudios se observó una variedad en el predominio de nivel de autoestima y estilos de vida ${ }^{12,13}$. En el estudio la autoestima se manifestó alta con predominio en mujeres mostrándose autoestima baja en el sexo masculino; esta discrepancia puede ser referida por diversos factores que no fueron tomados en cuenta en el presente estudio como: el nivel educativo, evolución de la enfermedad y nivel socioeconómico.

En un estudio que tuvo por objetivo evaluar las variables psicológicas en pacientes con DM2 también obtuvieron como resultado un autoestima alta o favorable sin embargo la mayor parte de la muestra estudiada se caracterizó por presentar un estilo de vida poco favorable y desfavorable ${ }^{13}$; este resultado es alarmante ya que la frecuencia de personas que poseen una autoestima alta mantienen estilos de vida desfavorables, lo que propicia el desarrollo de la enfermedad. Por lo que estadísticamente no resulto una correlación significativa entre las variables: estilo de vida y autoestima; esta relación puede tiene su explicación en que las personas con DM2 a pesar de tener autoestima alta pueden tener mínima o nula percepción sobre las consecuencias adyacentes hacia la enfermedad impidiendo llevar un control correcto hacia la DM2.

Finalmente, el estudio concluye que aunque no se asociaron las variables, la autoestima alta y media prevalecieron en la muestra, importantes para mantener el control de la enfermedad, por tanto es necesario incrementar el nivel de estilo de vida favorable.

En las dimensiones de estilos de vida y autoestima no se demostró asociación estadísticamente significativa entre las variables, sin embargo juegan un papel fundamental en el aspecto biopsicosocial de las personas con DM2 y sus familias. Respecto a los resultados del estudio hubo limitantes en la presente investigación ya que tal vez los resultados 
derivados dependan de otras variables que no fueron estudiadas como lo son: nivel socioeconómico, factores culturales, creencias y nivel educativo.

Estos resultados deben ser considerados por los profesionales de salud para desarrollar alternativas dirigidas a optimizar su estado físico, mental y emocional en función de una mejor calidad de vida, asimismo implementar programas de intervención en promoción a la salud para modificar los estilos de vida desfavorables presentes en las personas diagnosticadas con DM2 como a los que se encuentran en vulnerabilidad de padecerla.

\section{Agradecimientos:}

A la Jefatura de la UCIIS por lasfacilidades otorgadas para la realización del estudio, al personal docente de la unidad, a los estudiantes de VIII semestre de Licenciatura en Enfermería por su participación en la recolección de datos y de manera muy especial a los grupos de adultos mayores "Nueva llusión" y "Ganas de Vivir" por su entusiasta participación.

\section{4}




\section{REFERENCIAS BIBLIOGRÁFICAS}

1. Organización Mundial de la Salud. Informe sobre la situación mundial de las enfermedades no transmisibles 2010; 1-20. Disponible

2. Hernández Avila M, Gutiérrez JP, Reynoso Noverón N. Diabetes mellitus en México. El estado de la epidemia. Rev Salud Pública Méx x 2013; 55(2): 129- 136. Disponible en: http://bvs.insp.mx/rsp/articulos/articulo. php?id $=002844$

3. Gobierno de la Republica. Plan Nacional de Desarrollo 2013- 2018. Programa Sectorial de Salud. 1-18 Disponible en: http://Www.salud.gob.mx/indicadores 1378/pdf/programa.pdf

4. Encuesta Nacional de Salud y Nutrición 2012. Evidencia para la política pública en salud. 2012; 1-4. Disponible en: http://ensanut.insp. $m x /$ doctos/analiticos/DiabetesMellitus.pdf

5. Medellín-Vélez $B$. Desarrollo de capacidades de autocuidado en personas con diabetes mellitus tipo 2. Rev Enferm Inst Mex Seguro Soc 2007; 15 (2):91-98. Disponible en: http://www.medigraphic.com/pdfs/ enfermeriaimss/eim-2007/eim072f.pdf

6. Ramírez OM, Ascanio CM, Coronado CM, Gómez MC, Mayorga AL, Medina OR, et al. Estilo de vida actual de los pacientes con diabetes mellitus tipo 2. Rev0 Ciencia y Cuidado. 2011; 8(1): 21-8.

Disponible en: http://dialnet.unirioja.es/servlet/articulo ? codigo $=3853505$

7. Federación Internacional de Diabetes. Atlas de la diabetes de la FID. 6» ed. 2013. Version online.

Disponible en: http://www.idf.org/diabetesatlas

8. Salazar-Estrada JG, Torres-López TM, ColungaRodríguez C, Angel- González M. Calidad de vida, salud y trabajo la relación con diabetes mellitus tipo 2. Rev Salud Pública y Nutrición. 2009; 10(3): 1-10. Disponible en: http://www.respyn.uanl.mx/x/3/articulos/cal_vida_diabmellitus.htm
9. Rosenberg, M. Conceiving the self. New York: Basic Books; 1979.

10. López-Carmona, JM, Ariza-Andraca, CR, Rodríguez - Moctezuma, JR y Munguía-Miranda, C. (2003). Construcción y validación inicial de un instrumento para medir el estilo de vida en pacientes con diabetes mellitus tipo 2. Salud pública de México. 45 (4): 43-51.

11. Martín Albo J, Núñez JL, Navarro JG, Grijalvo F. The Rosenberg Self-Esteem Scale: Translation and Validation in University Students. J Psych. 2007; 10(2): $458 f i 467$.

12. Colunga-Rodríguez $C$, García de Alba JE, Angel - González M, Salazar-Estrada JG. Caracterización psicosocial de pacientes con diabetes tipo 2 en atención primaria. Rev Cubana Salud Pública 2008; 34(4): 1-6.

Disponible en: $h t t p: / / s c i e l o . s / d . c u / s c i e l o . p h p ? p i d=S 086$ 434662008000400005\&script=sci_arttext\&tlng=pt

13. Martínez-Brito $\bigcirc$, Camarero-Fortaleza $\bigcirc$, Carrio -Urra J, Bordón-Valdivia M. Comportamiento de variables psicológicas en pacientes con Diabetes Mellitus tipo II. 2009; 57-67.

Disponible en: http://www.cpicmha.sld.cu/hab/pdf/ vol77_7_77/hab08777.pdf

14. Gomes-Villas Boas LC, Foss MC, Freitas MCF, Pace AE. Relación entre apoyo social, adhesión al tratamiento y control metabólico de personas con Diabetes Mellitus. Rev. Latino-Am. Enfermagem, 201 1; 20(1): 1-8.

Disponible en: http://www.scielo.br/pdf/rlae/v20n 1/ es_08.pdf

15. Ballesteros-Jiménez S. Gerontología. Un saber multidisciplinar. (Editorial) Madrid (España): Universidad Nacional de Educación a Distancia; 2004. 
Cuadro I. Nivel de estilo de vida de los pacientes con Diabetes Mellitus tipo 2 de una comunidad suburbana. Delegación Villa de Pozos, SLP. Febrero-Abril de 2014 n=81

\begin{tabular}{|c|c|c|c|c|}
\hline Estilo de vida & I & Fr. & 1 & $\%$ \\
\hline Desfavorable & I & 3 & I & 3.7 \\
\hline Poco favorable & I & 45 & I & 55.6 \\
\hline Favorable & I & 33 & I & 40.7 \\
\hline Total & 1 & 81 & I & 100 \\
\hline
\end{tabular}

Fuente directa

Cuadro II. Estilo de vida por indicador de los pacientes con Diabetes Mellitus tipo 2 de una comunidad suburbana. Delegación Villa de Pozos, SLP. Febrero-Abril de 2014

\begin{tabular}{|c|c|c|c|c|c|c|c|}
\hline \multirow[t]{2}{*}{ Indicador } & \multicolumn{2}{|c|}{ Favorable } & \multicolumn{2}{|c|}{ oco favorabl } & \multicolumn{2}{|c|}{ Desfavorable } & Total \\
\hline & Fr. & $\%$ & Fr. & $\%$ & Fr. & $\%$ & \\
\hline Nutrición & 18 & 22.2 & 34 & 41.9 & 29 & 35.8 & 81 \\
\hline Actividad física & 37 & 45.6 & 17 & 20.9 & 27 & 33.3 & 81 \\
\hline Toxicomanias & 21 & 25.9 & 13 & 16 & 47 & 58 & 81 \\
\hline $\begin{array}{l}\text { Relaciones con } \\
\text { familia y amigos }\end{array}$ & 35 & 43.2 & 21 & 25.9 & 25 & 30.8 & 81 \\
\hline Adherencias al tratamiento & 23 & 28.3 & 31 & 38.2 & 27 & 33.3 & 81 \\
\hline
\end{tabular}

Fuente directa

Cuadro IV. Nivel de autoestima de los pacientes con Diabetes Mellitus tipo 2 de una comunidad suburbana.Delegación Villa de Pozos, SLP. Febrero-Abril de 2014

\begin{tabular}{|l|cccc|}
\hline Nivel de autoestima & | & Fr. & | & $\%$ \\
\hline Baja & | & 11 & $\mid$ & 13.5 \\
\hline Media & | & 19 & $\mid$ & 23.4 \\
\hline Alta & | & 51 & $\mid$ & 62.9 \\
\hline Total & | & 81 & $\mid$ & 100 \\
\hline
\end{tabular}

Fuente directa 